\title{
Fixed-pressure CPAP versus auto-adjusting CPAP: comparison of efficacy on blood pressure in obstructive sleep apnoea, a randomised clinical trial
}

\author{
J L Pépin, ${ }^{1,2}$ R Tamisier, ${ }^{1,2}{ }^{2}$ J P Baguet, ${ }^{6}$ B Lepaulle, ${ }^{4}$ F Arbib, $^{4} \mathrm{~N}$ Arnol, ${ }^{1,2}$ \\ J F Timsit, ${ }^{5}$ P Lévy ${ }^{1,2}$
}

${ }^{1}$ University of Grenoble Alpes, HP2; Inserm, U1042,

Grenoble, France

${ }^{2} \mathrm{CHU}$ de Grenoble, Laboratoire EFCR, Pôle THORAX et VAISSEAUX, Grenoble, France ${ }^{3} \mathrm{CHU}$ de Grenoble, Clinique de Cardiologie, Grenoble, France

${ }^{4}$ Cabinet de Pneumologie privé, Echirolles, France ${ }^{5}$ IAME UMR 1137 Inserm Université Paris Diderot, F-75018, Paris, France ${ }^{6}$ Groupe Hospitalier Mutualiste de Grenoble, Clinique de Cardiologie, Grenoble, France

\section{Correspondence to} Professor Jean Louis Pépin Laboratoire EFCR, CHU de Grenoble, BP217X 38043 Grenoble cedex 09, France; jpepin@chu-grenoble.fr

$J L P$ and RT are co-first authors. JFT and PL are co-senior authors.

Received 16 August 2015 Revised 27 February 2016 Accepted 17 March 2016 Published Online First 18 April 2016

\section{ABSTRACT}

Background Millions of individuals with obstructive sleep apnoea (OSA) are treated by CPAP aimed at reducing blood pressure (BP) and thus cardiovascular risk. However, evidence is scarce concerning the impact of different CPAP modalities on BP evolution.

Methods This double-blind, randomised clinical trial of parallel groups of patients with OSA indicated for CPAP treatment compared the efficacy of fixed-pressure CPAP (FP-CPAP) with auto-adjusting CPAP (AutoCPAP) in reducing $B P$. The primary endpoint was the change in office systolic BP after 4 months. Secondary endpoints included $24 \mathrm{~h}$ BP measurements.

Results Patients (322) were randomised to FP-CPAP $(n=161)$ or AutoCPAP $(n=161)$. The mean apnoea +hypopnoea index (AHI) was 43/h (SD, 21); mean age was 57 (SD, 11), with $70 \%$ of males; mean body mass index was $31.3 \mathrm{~kg} / \mathrm{m}^{2}(\mathrm{SD}, 6.6)$ and median device use was $5.1 \mathrm{~h} /$ night. In the intention-to-treat analysis, office systolic blood pressure decreased by $2.2 \mathrm{~mm} \mathrm{Hg}(95 \% \mathrm{Cl}$ -5.8 to 1.4$)$ and $0.4 \mathrm{~mm} \mathrm{Hg}(-4.3$ to 3.4$)$ in the FPCPAP and AutoCPAP group, respectively (group difference: $-1.3 \mathrm{~mm} \mathrm{Hg}(95 \% \mathrm{Cl}-4.1$ to 1.5$) ; p=0.37$, adjusted for baseline BP values). $24 \mathrm{~h}$ diastolic BP (DBP) decreased by $1.7 \mathrm{~mm} \mathrm{Hg}(95 \% \mathrm{Cl}-3.9$ to 0.5$)$ and $0.5 \mathrm{~mm} \mathrm{Hg}(95 \% \mathrm{Cl}-2.3$ to 1.3$)$ in the FP-CPAP and AutoCPAP group, respectively (group difference: $-1.4 \mathrm{~mm} \mathrm{Hg}(95 \% \mathrm{Cl}-2.7$ to -0.01$) ; p=0.048$, adjusted for baseline BP values).

Conclusions The result was negative regarding the primary outcome of office BP, while FP-CPAP was more effective in reducing $24 \mathrm{~h}$ DBP (a secondary outcome). Trial registration number NCT01090297.

\section{INTRODUCTION}

Obstructive sleep apnoea (OSA) is characterised by recurrent pharyngeal collapses occurring during sleep. OSA is a chronic disease, as frequent as asthma, affecting 5\%-15\% of the general population, and steadily worsens up to age $60 .^{12}$ OSA has a dose-response relationship with hypertension. ${ }^{3} 4$ Hypertension is among the factors that contribute to cardiovascular morbidity and mortality in OSA, ${ }^{5}{ }^{6}$ increasing the risk of fatal and non-fatal cardiovascular events, ${ }^{5}$ such that OSA represents an independent risk factor for sudden cardiovascular death. ${ }^{7}$

CPAP remains the first-line therapy for OSA. Randomised controlled trials (RCTs) and

\section{Key messages}

What is the key question?

- Is fixed pressure CPAP superior to autoadjusting CPAP in reducing blood pressure in patients with obstructive sleep apnoea (OSA)?

\section{What is the bottom line?}

- In this double-blind, randomised clinical trial of parallel groups involving 322 patients with OSA indicated for CPAP treatment, although fixed pressure and auto-adjusting CPAP had similar impact on clinical blood pressure (primary outcome), fixed pressure CPAP was more effective than auto-adjusting CPAP in reducing $24 \mathrm{~h}$ diastolic blood pressure (secondary outcome).

\section{Why read on?}

- The interest of fixed pressure CPAP for reducing $24 \mathrm{~h}$ blood pressure might be of particular clinical relevance for patients with OSA, with poorly controlled or resistant hypertension.

meta-analyses have established the efficacy of CPAP in reducing sleepiness and improving daytime functioning. ${ }^{8-11}$ Regarding cardiovascular outcomes, meta-analyses have demonstrated that CPAP reduces $24 \mathrm{~h}$ mean blood pressure (BP) by approximately $2 \mathrm{~mm} \mathrm{Hg}$ (pooled estimated effect), ${ }^{12-17}$ the extent of improvement being larger in the subgroup with resistant hypertension. ${ }^{18}$ The intention-totreat (ITT) analysis of the largest RCT conducted to date failed to show any significant effect of CPAP on incident hypertension and cardiovascular events, but showed a significant effect for the subgroup of patients with OSA using CPAP for more than $4 \mathrm{~h}$ a day. ${ }^{19}$ There is high heterogeneity in $\mathrm{BP}$ responses to CPAP, depending on OSA severity, nightly use of $\mathrm{CPAP}^{2021}$ and hypertensive status. Among factors influencing BP response to CPAP, there is little data regarding the role of CPAP treatment modalities; only a small sample-sized RCT suggests that fixed-pressure CPAP (FP-CPAP) is superior to auto-adjusting CPAP (AutoCPAP) vis-à-vis improvement in office $\mathrm{BP}^{22}$

AutoCPAP changes the pressure delivered depending on the residual events detected at any 
given time, and applies the lowest effective pressure. ${ }^{23}$ The average overnight applied pressure is significantly lower with AutoCPAP, while comparably low apnoea+hypopnoea indices are achieved. Theoretically, when using AutoCPAP for long-term treatment, varying the pressure delivered would improve patient comfort and thus enhance compliance. However, the variations in pressure associated with the functioning of AutoCPAP devices may induce micro-arousals and potentially change sleep macrostructure in some patients. ${ }^{24} 25$ This could lead to greater sympathetic activity that counters BP reduction in response to CPAP treatment.

Thus, while AutoCPAP devices are increasingly used ${ }^{26}$ for long-term treatment of OSA, the reduction in BP remains a key issue for improving cardiovascular risk in patients with OSA. A clear answer regarding the respective impact of FP-CPAP versus AutoCPAP on BP is needed. The objective of our study was to conduct a large RCT to compare the efficacy of FP-CPAP with AutoCPAP in reducing BP in patients with OSA.

\section{METHODS}

\section{Design overview}

The AgirSASadom study was a single-site randomised doubleblind, parallel, 4-month controlled trial. The study was designed to assess whether FP-CPAP was superior to AutoCPAP in reducing $\mathrm{BP}$ in newly diagnosed patients with OSA. The trial was approved by the appropriate ethical committee, Comité de Protection des Personnes Sud-Est V, Grenoble, FRANCE (REC No: 09-AGIR-2) and registered on clinicaltrials.com (NCT01090297). All participants provided signed informed consent to participate in the study.

\section{Participants}

Patients referred to sleep clinics, usually due to daytime sleepiness, snoring, witnessed apneas or nocturia were assessed for eligibility. The inclusion criteria were patients with OSA aged 18-80 years with a recommendation to commence CPAP therapy. The exclusion criteria were pregnancy, cardiac failure and central sleep apnoea defined by a central apnoea index above 5/h. Patients were recruited from June 2010 and follow-up completed by October 2012. We should report one serious unintended event in one patient in the FP-CPAP group who presented a stroke during the protocol.

\section{Procedures}

Sleep studies

All patients were diagnosed with OSA using overnight respiratory polygraphy (PG) or polysomnography (PSG).

For polysomnography, continuous recordings were taken with electrode positions $\mathrm{C} 3 / \mathrm{A} 2-\mathrm{C} 4 / \mathrm{A} 1-\mathrm{Cz} / 01$ of the international 10-20 Electrode Placement System, eye movements, chin electromyogram and ECG with a modified V2 lead. Sleep was scored manually according to standard criteria. ${ }^{27}$ Cardiorespiratory polygraphy included at least the following signals: nasal pressure, thoracic and abdominal movements, $\mathrm{SaO}_{2}$, and body position. For polysomnography and polygraphy, airflow was measured with nasal pressure prongs, together with the sum of oral and nasal thermistor signals. Respiratory effort was monitored using abdominal and thoracic bands. Oxygen saturation was measured using a pulse oximeter. An apnoea was defined as the complete cessation of airflow for at least $10 \mathrm{~s}$ and hypopnoea as a reduction of at least $50 \%$ in the nasal pressure signal or a decrease of between $30 \%$ and $50 \%$ associated with either oxygen desaturation of at least $3 \%$ or an EEG arousal, ${ }^{27}$ both lasting for at least $10 \mathrm{~s}$. Apnoea was classified as obstructive, central or mixed, according to the presence or absence of respiratory efforts. The classification of hypopnoea as obstructive or central was based on the thoracoabdominal band signal and the shape of the respiratory nasal pressure curve (flow limited aspect or not). The apnoea+hypopnoea index (AHI), defined as the number of apnoea and hypopnoea per hour of sleep (full polysomnography) or per hour of recording (polygraphy without EEG recording), was calculated. One hundred and twenty-seven patients had full PSG and 195 had respiratory polygraphy. The patients with PSG/PG were homogeneously distributed between the two arms, as expected for a large RCT $\left(\chi^{2}: \mathrm{p}=0.57\right)$.

\section{BP measurements}

Clinical BP was measured by mercury sphygmomanometer on three occasions in line with the European Society of Hypertension-European Society of Cardiology guidelines. $^{28}$ Systolic BP (SBP) and diastolic BP (DBP) were assessed. Mean arterial BP (MABP) was calculated as $\mathrm{DBP}+1 / 3(\mathrm{SBP}-\mathrm{DBP})$.

Ambulatory blood pressure monitoring (ABPM) was carried out with Spacelabs 90207 devices (Spacelabs International, Redmond, Washington, USA). The measurements were made every $15 \mathrm{~min}$ in the day and every $30 \mathrm{~min}$ at night. The following ABPM parameters were studied: mean SBP, DBP and HR over $24 \mathrm{~h}$ and during daytime (07:00-22:00) and nighttime (22:00-07:00). The summary values in the ABPM report for each patient in the data analysis were used. ${ }^{18}$ This was an average taken by subject and recording session (baseline/ 4 months). Data relating to the average daytime and nighttime SBP, DBP and mean BP were recorded. Values of SBP $>260 \mathrm{~mm} \mathrm{Hg}$ or $<70 \mathrm{~mm} \mathrm{Hg}$ and DBP $>150 \mathrm{~mm} \mathrm{Hg}$ or $<40 \mathrm{~mm} \mathrm{Hg}$ were automatically eliminated. Daytime hypertension was defined as daytime SBP $>135 \mathrm{~mm} \mathrm{Hg}$ and/or DBP $>85 \mathrm{~mm} \mathrm{Hg}$, and nighttime hypertension as nighttime SBP $>120 \mathrm{~mm} \mathrm{Hg}$ and/or DBP > $70 \mathrm{~mm} \mathrm{Hg} .^{28}$

\section{Carotid-femoral pulse wave velocity measurements}

A one-pulse transducer was placed on the skin over the right common carotid and common femoral artery, with the patient in a supine position, and after at least $15 \mathrm{~min}$ of rest. The time delay of simultaneously recorded pulse waves between the carotid and the thigh was measured with a Complior device (Artech Medical; Pantin, France) and averaged over 10 consecutive cycles. The carotid-femoral pulse wave velocity (PWV) was calculated as the distance between the arterial sites divided by the time delay. The mean value of three consecutive measurements in each subject was used for analysis.

\section{Randomisation and interventions}

Patients meeting eligibility criteria were randomised to either FP-CPAP or AutoCPAP treatment. Randomisation was conducted by telephone call to a clinical trials statistician, independent of the study using a computer-generated random numbers list (six patients per block). In both arms, patients were randomly allocated to three different brands of the devices (Philips, Resmed and Weinman). Optimal CPAP pressure was titrated during eight nights at home using an AutoCPAP device (RESMED) to obtain a FP-CPAP value. The optimal pressure (95th percentile) was determined by one expert researcher, based on visual evaluation of the raw data recordings from nights with no significant leaks (less than $0.40 \mathrm{~L} / \mathrm{s}$ ). The mean CPAP pressure in the FP-CPAP arm was $9.3 \mathrm{~mm} \mathrm{Hg}$ (SD: $1.9 \mathrm{~mm} \mathrm{Hg}$; median: $9.0 \mathrm{~mm} \mathrm{Hg}$ ). This fixed pressure was then maintained throughout the study in patients assigned to the 
FP-CPAP group. In the AutoCPAP group, the window for pressure variation was a minimum interval of $5 \mathrm{~cm} \mathrm{H}_{2} \mathrm{O}$. All patients, investigators and outcome assessment technicians were blinded to the arms to which the patients were allocated.

\section{Main outcome measures}

All outcome measures were made on two occasions, at baseline prior to treatment and then at the end of the 4 months' treatment. The primary outcome was the change in office SBP between baseline and 4 months of FP-CPAP or AutoCPAP. Secondary cardiovascular outcomes included change in $24 \mathrm{~h}$ ambulatory BP and arterial stiffness. ${ }^{29}$

\section{Statistical analysis}

Continuous variables are expressed as median (25th/75th percentiles) or mean (SD), while categorical variables are reported as absolute numbers and percentages. Baseline comparisons between groups were made using Student's t test or MannWhitney U test, depending on the validation of normal distribution. For discrete variables, a $\chi^{2}$ test was used. Normality was assessed using the Shapiro-Wilk test. All randomised patients were included in the ITT analysis. The per-protocol (PP) population was defined as patients who completed the fourth month visit without any protocol deviation.

We powered the study based on an office BP outcome. A previous study showed that OSA treatment with FP-CPAP lowered systolic BP by $6 \mathrm{~mm} \mathrm{Hg}$ relative to AutoCPAP. ${ }^{22}$ We assumed the impact of CPAP to be $6 \mathrm{~mm} \mathrm{Hg}^{22}$ in the FP-CPAP group and $0 \mathrm{~mm} \mathrm{Hg}$ in the AutoCPAP group (SD 15). Assuming an $\alpha$ error of $5 \%$ and a statistical power of $90 \%, 161$ patients per arm needed to be enrolled in the study. An interim analysis was carried out when 150 patients had completed the study. The Peto group's sequential stopping method was used to preserve the intended $\alpha$ level and power by adopting stringent criteria (low nominal $\mathrm{p}$ values $(\mathrm{p}<0.001)$ ) during the interim analyses. ${ }^{30}$ The interim analysis was defined a priori, validated by the ethics committee and included in the registration of the clinical trial. The intragroup differences from the beginning to the end of the study were evaluated with a paired $t$ test. Unadjusted treatment effect and baseline BP adjusted treatment effect were estimated respectively by a mixed model with two factors (fixed factor: group; random factor: time) and by analysis of variance including baseline BP. In ITT analysis, missing data were replaced by multiple imputations using a regression model. We used a multiple imputation procedure that creates multiple imputed data sets with a regression method. ${ }^{31}$ Sequential regression models (from the least missing to the most missing variable) were created. This method assumes that the data are from a multivariate normal distribution; thus, when normality was not observed, variables were log-transformed before the imputation process and then were reverse-transformed to create the imputed data set. To guarantee the general validity of analyses of the resultant multiple imputed data set, we included as many variables as we could in the regression models. ${ }^{32}$

We had no missing data for clinical BP at baseline. For multiple imputations of BP at 4 months, we included baseline values of office BP, anthropometric parameters (age, body mass index, sex and waist circumference), medications, biological parameters, additional BP values, sleep apnoea parameters at baseline and under-treatment. Missing data for $24 \mathrm{~h} \mathrm{BP}$ measurements have been imputed using the same methodology and by adding clinical BP values.

Data management and statistical analyses were performed using SAS (V.9.2, OSA Institute, Cary, North Carolina, USA).

\section{RESULTS}

Of the initial 569 recruited patients, 322 were randomised, 161 to the FP-CPAP group and 161 to AutoCPAP treatment (ITT population) (figure 1). The middle-aged, obese, predominantly male and hypertensive study population reflected patients with OSA, usually seen in clinical practice (table 1). For the whole study population (table 2 ), the median (25th/75th percentiles) baseline $24 \mathrm{~h}$ mean BP was $94.0(89.0 / 99.3) \mathrm{mm} \mathrm{Hg}$; SBP, 128.0 (120.0/136.0) $\mathrm{mm} \mathrm{Hg}$ and DBP, 78.0 (73.0/83.0) $\mathrm{mm} \mathrm{Hg}$. CPAP compliance did not differ between the groups with median (25th/75th percentiles) CPAP use of $5.2(3.4 / 6.5)$ and 5.1 (3.5/ 6.6) hours per night in the FP-CPAP and AutoCPAP arms, respectively. The percentage of patients with OSA using CPAP more than $4 \mathrm{~h} /$ night was $64.6 \%$ and $66.5 \%$ in the FP-CPAP and AutoCPAP arms, respectively. The median (25th/75th percentiles) residual AHI following the application of CPAP was $3.6(2.1 / 6.9)$ events/h without difference between groups (AutoCPAP: $3.6 \quad(2.2 / 6.5)$ and FP-CPAP: $3.6 \quad(2.0 / 7.9)$ respectively).

\section{ITT analysis}

When the changes in BP from baseline to 4 months were compared between study groups by ITT, the office systolic BP decreased by $2.2 \mathrm{~mm} \mathrm{Hg}(95 \% \mathrm{CI}-5.8$ to 1.4$)$ in the FP-CPAP group and by $0.4 \mathrm{~mm} \mathrm{Hg}(95 \% \mathrm{CI}-4.3$ to 3.4$)$ in the AutoCPAP group (difference between groups: $-1.3 \mathrm{~mm} \mathrm{Hg}$ (95\% CI -4.1 to 1.5 ); $\mathrm{p}=0.37$, adjusted for baseline BP values and history of hypertension) (table 3). From baseline to 4 months, $24 \mathrm{~h}$ DBP decreased by $1.7 \mathrm{~mm} \mathrm{Hg}(95 \% \mathrm{CI}-3.9$ to $0.5)$ in the FP-CPAP group and by $0.5 \mathrm{~mm} \mathrm{Hg}(95 \% \mathrm{CI}-2.3$ to 1.3 ) in the AutoCPAP group (difference between groups: $-1.4 \mathrm{~mm} \mathrm{Hg}$ (95\% CI -2.7 to -0.01$) ; \mathrm{p}=0.048$, adjusted for baseline BP values and history of hypertension). In both arms, the prevalence of non-dipping status was unchanged after the intervention.

\section{PP analysis}

In a PP analysis (133 patients in the FP-CPAP group; 143 patients in the AutoCPAP group), patients in the FP-CPAP group showed a statistically significant decrease of $1.3 \mathrm{~mm} \mathrm{Hg}(95 \%$ CI 2.4 to 0.1$), \mathrm{p}=0.032$ in $24 \mathrm{~h} \mathrm{DBP}$ and $1.6 \mathrm{~mm} \mathrm{Hg}$ (95\% CI 3.1 to 0.01 ), $\mathrm{p}=0.049$ in $24 \mathrm{~h}$ mean daytime $\mathrm{BP}$ (table 4 and figure 2). Graphically, it can be clearly seen (figure 2) that the range of decrease is greater with FP-CPAP when compared with AutoCPAP.

A direct comparison of the two CPAP arms restricted to the subgroup of good compliers ( $>4 \mathrm{~h} /$ night) did not demonstrate a superiority of one CPAP modality, both in ITT and PP analyses. Improvement in quality of life as assessed by SF12 was the same in the two arms.

\section{DISCUSSION}

Hypertension affects about $25 \%$ of the adult population worldwide. It ranks as the leading chronic risk factor for mortality, accounting for $13.5 \%$ of all deaths. Half of all strokes and ischaemic heart disease events are attributable to high $\mathrm{BP}^{33} 34$ OSA is now recognised as a risk factor for the development of hypertension in European and US International Guidelines. After accounting for the main confounders, OSA and hypertension remain linked in a dose-response fashion. ${ }^{3}$ In a large prospective observational cohort followed for more than 12 years, it was shown that compared with controls, the adjusted HRs for incident hypertension were greater among patients with severe 


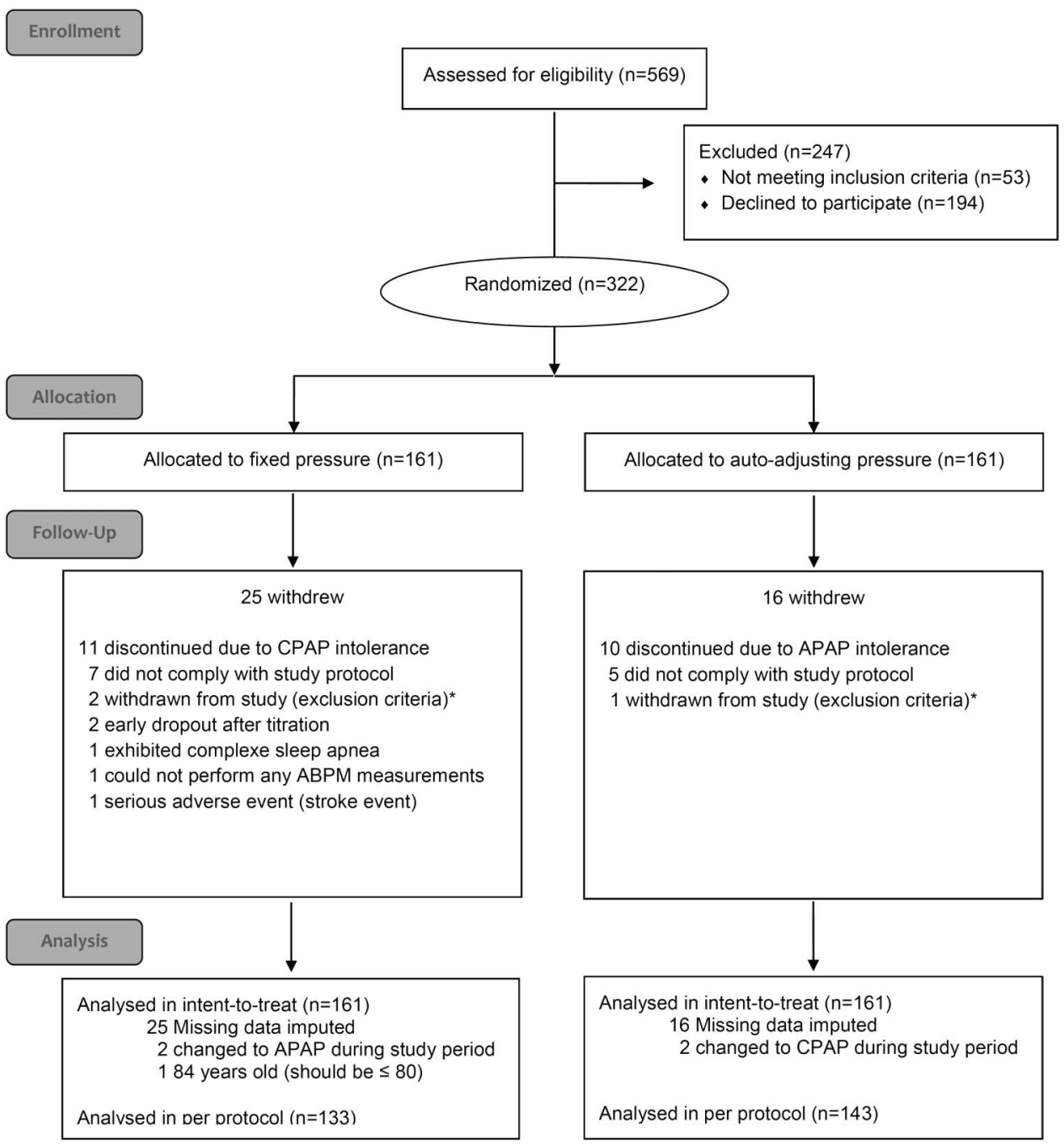

Figure 1 Flow diagram showing the trial allocation.

OSA who declined CPAP therapy and among those nonadherent to CPAP therapy, whereas the HR was lower in patients with OSA who were treated with CPAP therapy for more than $4 \mathrm{~h} /$ night. $^{35}$ This suggests that treating OSA could be an important step towards reducing BP and thus cardiovascular risk. Meta-analyses of RCTs have demonstrated that CPAP reduces $24 \mathrm{~h}$ mean $\mathrm{BP}$ by approximately $-2 \mathrm{~mm} \mathrm{Hg}$ (pooled estimated effect). ${ }^{12-17}$ In these meta-analyses, heterogeneity is a clear concern. Also, Bratton et $a l^{20}$ found no beneficial effect on $\mathrm{BP}$ in patients with minimally symptomatic OSA, except in those patients who used CPAP for $>4 \mathrm{~h} /$ night. $^{21}$ In summary, meta-analyses agree on the fact that $\mathrm{BP}$ reduction after CPAP initiation is of greater amplitude in patients with severe OSA and/or poorly controlled/resistant hypertension ${ }^{18}$ and in patients

Table 1 Baseline characteristics of all randomised patients

\begin{tabular}{|c|c|c|c|c|}
\hline & All patients, $n=322$ & AutoCPAP group, $n=161$ & FP-CPAP group, $n=161$ & p Value \\
\hline BMI & $30.0(26.9 / 34.5)$ & $29.4(26.6 / 34.3)$ & $30.8(27.1 / 34.6)$ & 0.25 \\
\hline Age, years & $58(51 / 65)$ & $58(49 / 64)$ & $58(52 / 66)$ & 0.30 \\
\hline Men, \% & 70.2 & 70.80 & 69.60 & 0.81 \\
\hline Current smoker or ex-smoker, \% & 60.20 & 59.00 & 61.50 & 0.65 \\
\hline Apnoea-hypopnoea index, event/h & $38.8(28.4 / 56.0)$ & $37.7(28.4 / 54.5)$ & $39.0(28.4 / 56.9)$ & 0.43 \\
\hline Mean nocturnal $\mathrm{SaO}_{2}, \%$ & $92.8 \pm 2.9$ & $92.9 \pm 3.0$ & $92.7 \pm 2.8$ & 0.62 \\
\hline Epworth Sleepiness Scale & $9(6 / 14)$ & $9(6 / 13)$ & $10(6 / 14)$ & 0.10 \\
\hline Hypertensive, \% & 65.50 & 60.90 & 70.20 & 0.08 \\
\hline Diabetic, \% & 19.20 & 18.20 & 20.30 & 0.64 \\
\hline
\end{tabular}


Table 2 Baseline BP and arterial stiffness characteristics

\begin{tabular}{lccc}
\hline & All patients, $\mathrm{n}=\mathbf{3 2 2}$ & AutoCPAP group, $\mathrm{n}=\mathbf{1 6 1}$ & FP-CPAP group, $\mathrm{n}=161$ \\
\hline Office SBP, mm Hg & $132.5(124.0 / 145.0)$ & $130.0(123.0 / 142.5)$ & $135.0(125.0 / 145.0)$ \\
Office DBP, mm Hg & $80.0(73.0 / 86.0)$ & $80.0(73.0 / 87.0)$ & $79.0(73.0 / 85.0)$ \\
$24 \mathrm{~h} \mathrm{SBP}, \mathrm{mm} \mathrm{Hg}$ & $128.0(120.0 / 136.0)$ & $126.0(118.0 / 135.0)$ & $128.8(121.0 / 136.0)$ \\
$24 \mathrm{~h} \mathrm{DBP}, \mathrm{mm} \mathrm{Hg}$ & $78.0(73.0 / 83.0)$ & $78.0(73.0 / 83.0)$ & $78.0(72.0 / 83.0)$ \\
$24 \mathrm{~h}$ mean BP, mm Hg & $94.0(89.0 / 99.3)$ & $94.0(89.0 / 98.0)$ & $94.0(89.0 / 101.0)$ \\
Daytime SBP, mm Hg & $132.0(124.0 / 141.0)$ & $131.0(123.0 / 141.0)$ & $133.0(125.0 / 142.0)$ \\
Daytime DBP, mm Hg & $82.0(76.0 / 88.0)$ & $82.0(76.0 / 88.8)$ & $82.0(75.9 / 88.0)$ \\
Daytime mean BP, mm Hg & $98.0(92.0 / 104.0)$ & $98.0(92.0 / 103.0)$ & $98.0(93.0 / 105.0)$ \\
Nighttime SBP, mm Hg & $118.0(109.0 / 127.2)$ & $118.0(108.0 / 128.0)$ & $119.0(110.0 / 127.0)$ \\
Nighttime DBP, mm Hg & $71.0(65.0 / 76.0)$ & $70.0(65.0 / 76.0)$ & $71.0(66.0 / 77.0)$ \\
Nighttime mean BP, mm Hg & $87.0(81.0 / 93.0)$ & $86.0(82.0 / 93.0)$ & $87.0(81.0 / 93.0)$ \\
Arterial stiffness, m/s & $10.3(9.2 / 11.6)$ & $10.3(9.2 / 11.6)$ & $10.2(9.2 / 11.5)$ \\
\hline
\end{tabular}

Data are presented as median (25th/75th percentiles).

AutoCPAP, auto-adjusted CPAP; BP, blood pressure; DBP, diastolic blood pressure; FP-CPAP, fixed-pressure CPAP; SBP, systolic blood pressure.

who comply with CPAP treatment. Surprisingly, the impact of different CPAP modalities on BP has been little studied. Our study, comparing FP-CPAP with AutoCPAP, was negative regarding its primary outcome of office BP, while FP-CPAP was more effective in reducing $24 \mathrm{~h}$ DBP (a secondary outcome).

Three RCTs have assessed changes in BP induced by FP-CPAP versus AutoCPAP.22 3637 Two studies did not find significant differences in BP changes between the two groups. ${ }^{36} 38{ }^{39}$ In these two studies, BP was a secondary outcome and, as stated by the authors themselves, the studies were not powered to look at the changes in BP with CPAP treatment. ${ }^{38}$ In the third study, ${ }^{22}$ significant reductions in office BP were observed in the FP-CPAP group but not in the AutoCPAP group. Ip et al, ${ }^{39}$ using data reported by Patruno et al, ${ }^{22}$ estimated a nearly significant greater reduction in SBP $(6 \mathrm{~mm} \mathrm{Hg}(95 \% \mathrm{CI}-1$ to $13 \mathrm{~mm} \mathrm{Hg})$; $\mathrm{p}=0.09)$ and a significant greater reduction in DBP $(8 \mathrm{~mm} \mathrm{Hg}$ (95\% CI 4 to $11 \mathrm{~mm} \mathrm{Hg}$ ); $\mathrm{p}<0.001$ ) with FP-CPAP compared with AutoCPAP. We used office SBP as the outcome in our study as it was the only significant outcome in the Patruno study and has previously been frequently used in studies comparing CPAP with an inactive control (44 studies in the meta-analysis by Bratton $\left.e t a l^{17}\right)$. This outcome is relevant as it is related to mortality and the incidence of late cardiovascular events. ${ }^{40}$ However, given the relatively small number of subjects in previous studies, these findings needed to be re-evaluated in a more highly powered study that also included $24 \mathrm{~h} \mathrm{BP}$ monitoring. Our study was clearly negative regarding the primary outcome, and the difference between the two CPAP modalities (1.4 $\mathrm{mm} \mathrm{Hg}$ (CI 95\% 2.7 to 0.01)) might appear to be of limited clinical relevance. However, even small effects on BP in the range of 1-2 $\mathrm{mm} \mathrm{Hg}$ are of clinical significance as they are associated with reduced odds of cardiovascular and cerebrovascular events. ${ }^{204041}$ There is increasing evidence that mean nocturnal BP is a major indicator of cardiovascular morbidity and mortality, irrespective of clinical $\mathrm{BP}^{42}$ The $24 \mathrm{~h}$ BP measurements are more sensitive to therapeutic interventions and provide specific prognostic information. This is particularly true in sleep apnoea-related hypertension. ${ }^{18} 4344$ While negative in terms of the primary outcome, our study provides interesting insights regarding $24 \mathrm{~h} \mathrm{ABPM}$ that might be of clinical relevance and a strong rationale for further studies.

One can hypothesise on the mechanisms potentially underlying the different impacts on BP of the two treatment modalities. Sleep fragmentation and alterations in sleep quality are

Table 3 Effect of CPAP treatments on BP levels in the intention-to-treat population

\begin{tabular}{|c|c|c|c|c|c|c|c|c|c|c|}
\hline \multirow[b]{2}{*}{ BP, mm Hg } & \multicolumn{3}{|c|}{ FP-CPAP $(n=161)$} & \multicolumn{3}{|c|}{ AutoCPAP $(n=161)$} & \multicolumn{2}{|l|}{ Intergroup raw } & \multicolumn{2}{|l|}{ Intergroup adjusted* } \\
\hline & Baseline & Follow-up & $\mathrm{p}$ Value & Baseline & Follow-up & $\mathrm{p}$ Value & $\begin{array}{l}\text { Differences } \\
(95 \% \mathrm{Cl})\end{array}$ & $\mathrm{p}$ Value & Differences (95\% Cl) & $\mathrm{p}$ Value \\
\hline Office SBP & $135.8 \pm 16.8$ & $133.6 \pm 15.9$ & 0.23 & $134.8 \pm 17.6$ & $134.3 \pm 17.7$ & 0.82 & $-1.7(-4.9$ to 1.4$)$ & 0.28 & $-1.3(-4.1$ to 1.5$)$ & 0.37 \\
\hline Office DBP & $79.6 \pm 9.8$ & $76.8 \pm 10.4$ & 0.014 & $80.2 \pm 9.7$ & $78.2 \pm 9.5$ & 0.06 & $-0.8(-2.9$ to 1.3$)$ & 0.44 & $-1.1(-2.9$ to 0.7$)$ & 0.25 \\
\hline $24 \mathrm{~h}$ SBP & $129.3 \pm 14.0$ & $127.2 \pm 14.0$ & 0.17 & $128.4 \pm 13.7$ & $128.1 \pm 14.2$ & 0.84 & $-1.8(-4.4$ to 0.8$)$ & 0.17 & $-1.5(-3.8$ to 0.8$)$ & 0.21 \\
\hline 24 h DBP & $78.6 \pm 8.8$ & $76.8 \pm 9.0$ & 0.08 & $78.6 \pm 8.3$ & $78.1 \pm 8.0$ & 0.59 & $-1.3(-2.7$ to 0.2$)$ & 0.08 & $-1.4(-2.7$ to -0.01$)$ & 0.048 \\
\hline $24 \mathrm{~h}$ mean BP & $95.3 \pm 9.2$ & $93.6 \pm 9.5$ & 0.10 & $94.9 \pm 8.9$ & $94.5 \pm 9.1$ & 0.72 & $-1.4(-3.2$ to 0.4$)$ & 0.13 & $-1.2(-2.9$ to 0.4$)$ & 0.13 \\
\hline Daytime SBP & $134.4 \pm 14.8$ & $132.4 \pm 14.6$ & 0.22 & $133.2 \pm 14.0$ & $132.9 \pm 14.4$ & 0.87 & $-1.8(-4.5$ to 1.0$)$ & 0.21 & $-1.3(-3.8$ to 1.2$)$ & 0.30 \\
\hline Daytime DBP & $82.4 \pm 9.7$ & $80.4 \pm 9.8$ & 0.06 & $82.5 \pm 8.7$ & $81.8 \pm 8.9$ & 0.47 & $-1.3(-3.1$ to 0.4$)$ & 0.13 & $-1.4(-3.0$ to 0.2$)$ & 0.09 \\
\hline Daytime mean BP & $99.3 \pm 10.0$ & $97.4 \pm 10.2$ & 0.08 & $98.8 \pm 8.9$ & $98.4 \pm 9.2$ & 0.69 & $-1.6(-3.5$ to 0.3$)$ & 0.11 & $-1.5(-3.2$ to 0.2$)$ & 0.09 \\
\hline Nighttime SBP & $119.9 \pm 14.7$ & $118.2 \pm 15.5$ & 0.31 & $119.6 \pm 15.8$ & $119.0 \pm 15.7$ & 0.72 & $-1.1(-3.9$ to 1.8$)$ & 0.46 & $-1.0(-3.6$ to 1.6$)$ & 0.45 \\
\hline Nighttime DBP & $71.4 \pm 8.9$ & $70.3 \pm 9.1$ & 0.26 & $71.3 \pm 9$ & $71.5 \pm 8.9$ & 0.85 & $-1.3(-3.0$ to 0.4$)$ & 0.13 & $-1.3(-2.8$ to 0.3$)$ & 0.10 \\
\hline Nighttime mean BP & $87.9 \pm 9.6$ & $86.9 \pm 10.1$ & 0.38 & $87.8 \pm 10.3$ & $87.6 \pm 10.0$ & 0.85 & -0.8 (-2.8 to 1.2$)$ & 0.45 & $-0.7(-2.5$ to 1.1$)$ & 0.42 \\
\hline
\end{tabular}


Table 4 Effect of CPAP treatments on BP levels in the per-protocol population

\begin{tabular}{|c|c|c|c|c|c|c|c|c|c|c|}
\hline \multirow[b]{2}{*}{$\mathrm{BP}, \mathrm{mm} \mathrm{Hg}$} & \multicolumn{3}{|c|}{ FP-CPAP $(n=133)$} & \multicolumn{3}{|c|}{ AutoCPAP $(n=143)$} & \multicolumn{2}{|l|}{ Intergroup raw } & \multicolumn{2}{|l|}{ Intergroup adjusted* } \\
\hline & Baseline & Follow-up & p Value & Baseline & Follow-up & p Value & Differences $(95 \% \mathrm{CI})$ & p Value & Differences $(95 \% \mathrm{Cl})$ & $p$ Value \\
\hline Office SBP & $136.0 \pm 15.4$ & $132.9 \pm 14.2$ & 0.007 & $134.6 \pm 17.1$ & $133.9 \pm 16.7$ & 0.52 & $-2.4(-5.5$ to 0.7$)$ & 0.13 & $-1.8(-4.6$ to 1.0$)$ & 0.20 \\
\hline Office DBP & $79.5 \pm 9.6$ & $76.1 \pm 9.3$ & $<0.001$ & $80.5 \pm 9.7$ & $78.0 \pm 8.9$ & 0.001 & $-1.0(-3.0$ to 1.0$)$ & 0.35 & $-1.4(-3.2$ to 0.3$)$ & 0.11 \\
\hline $24 \mathrm{~h} \mathrm{SBP}$ & $129.0 \pm 12.6$ & $126.8 \pm 11.4$ & 0.012 & $127.8 \pm 13.5$ & $127.6 \pm 12.9$ & 0.82 & $-2.0(-4.4$ to 0.3$)$ & 0.09 & $-1.7(-3.9$ to 0.5$)$ & 0.13 \\
\hline 24 h DBP & $78.8 \pm 8.2$ & $76.7 \pm 7.5$ & $<0.001$ & $78.6 \pm 8.2$ & $77.8 \pm 7.5$ & 0.08 & $-1.3(-2.6$ to -0.03$)$ & 0.046 & $-1.3(-2.4$ to -0.1$)$ & 0.032 \\
\hline $24 \mathrm{~h}$ mean BP & $95.4 \pm 8.2$ & $93.4 \pm 7.5$ & $<0.001$ & $94.8 \pm 8.8$ & $94.1 \pm 7.9$ & 0.24 & $-1.4(-2.9$ to 0.2$)$ & 0.08 & $-1.2(-2.5$ to 0.2$)$ & 0.09 \\
\hline Daytime SBP & $134.4 \pm 13.8$ & $131.8 \pm 11.9$ & 0.001 & $132.5 \pm 13.6$ & $132.5 \pm 12.9$ & 0.94 & $-2.5(-5.2$ to 0.2$)$ & 0.07 & $-1.8(-4.2$ to 0.5$)$ & 0.13 \\
\hline Daytime DBP & $82.8 \pm 9.0$ & $80.3 \pm 8.3$ & $<0.001$ & $82.5 \pm 8.5$ & $81.3 \pm 8.1$ & 0.031 & $-1.4(-2.8$ to 0.1$)$ & 0.07 & $-1.3(-2.6$ to 0.1$)$ & 0.07 \\
\hline Daytime mean BP & $99.6 \pm 9.2$ & $97.0 \pm 8.2$ & $<0.001$ & $98.6 \pm 8.9$ & $97.9 \pm 8.2$ & 0.28 & $-1.9(-3.6$ to -0.1$)$ & 0.041 & $-1.6(-3.1$ to -0.01$)$ & 0.049 \\
\hline Nighttime SBP & $119.3 \pm 12.6$ & $117.7 \pm 12.6$ & 0.12 & $119.3 \pm 15.3$ & $118.8 \pm 14.3$ & 0.58 & $-1.0(-3.6$ to 1.6$)$ & 0.45 & $-1.0(-3.4$ to 1.4$)$ & 0.40 \\
\hline Nighttime DBP & $71.4 \pm 7.9$ & $70.3 \pm 7.4$ & 0.040 & $71.5 \pm 9.0$ & $71.3 \pm 7.8$ & 0.81 & $-1.0(-2.6$ to 0.5$)$ & 0.19 & $-1.0(-2.4$ to 0.3$)$ & 0.13 \\
\hline Nighttime mean BP & $87.6 \pm 8.1$ & $86.6 \pm 8.0$ & 0.12 & $87.8 \pm 10.1$ & $87.4 \pm 8.9$ & 0.49 & $-0.6(-2.3$ to 1.2$)$ & 0.52 & $-0.6(-2.2$ to 0.9$)$ & 0.43 \\
\hline
\end{tabular}

associated with prevalent or incident hypertension, even if age, gender, environmental exposures and ethnic disparities are clear confounders. ${ }^{42}$ Any disturbance in sleep quality is a mechanism through which an increase in sympathetic tone and in turn elevated BP can appear. The manner in which AutoCPAP functions is associated with a more unstable upper airway that can sometimes induce micro-arousals. ${ }^{24}{ }^{25}$ Also, inappropriate increases in pressure can occur even in normal subjects after a change in body position, after a central apnoea, during a period of flow limitation or, most frequently, without any identifiable reason. ${ }^{45}$ There are potentially differences from one brand of AutoCPAP device to another. Devices differ in how sleep-disturbed breathing is detected and in how the operational algorithm responds. Accordingly, one of the strengths of our study was to have randomised to three different brands in the AutoCPAP arm. Thus, our results are not related to a particular brand. As stated above, acute increases in pressure can induce micro-arousals from sleep that have been associated with autonomic activation. Karasulu et $a l^{46}$ demonstrated that AutoCPAP devices did not improve heart rate variability, whereas FP-CPAP did. Also, AutoCPAP treatment was characterised by greater sympathetic activation and was associated with poorer cardiorespiratory coupling compared with FP-CPAP. ${ }^{47}$ Although our study did not aim to address these specific mechanisms, these figures provide a clear rationale towards explaining our results.

\section{Study limitations}

We adopted a clinical approach to setting up CPAP therapy, without preliminary in-laboratory CPAP titration. This might be considered as a limitation, but it reflects clinical practice and has
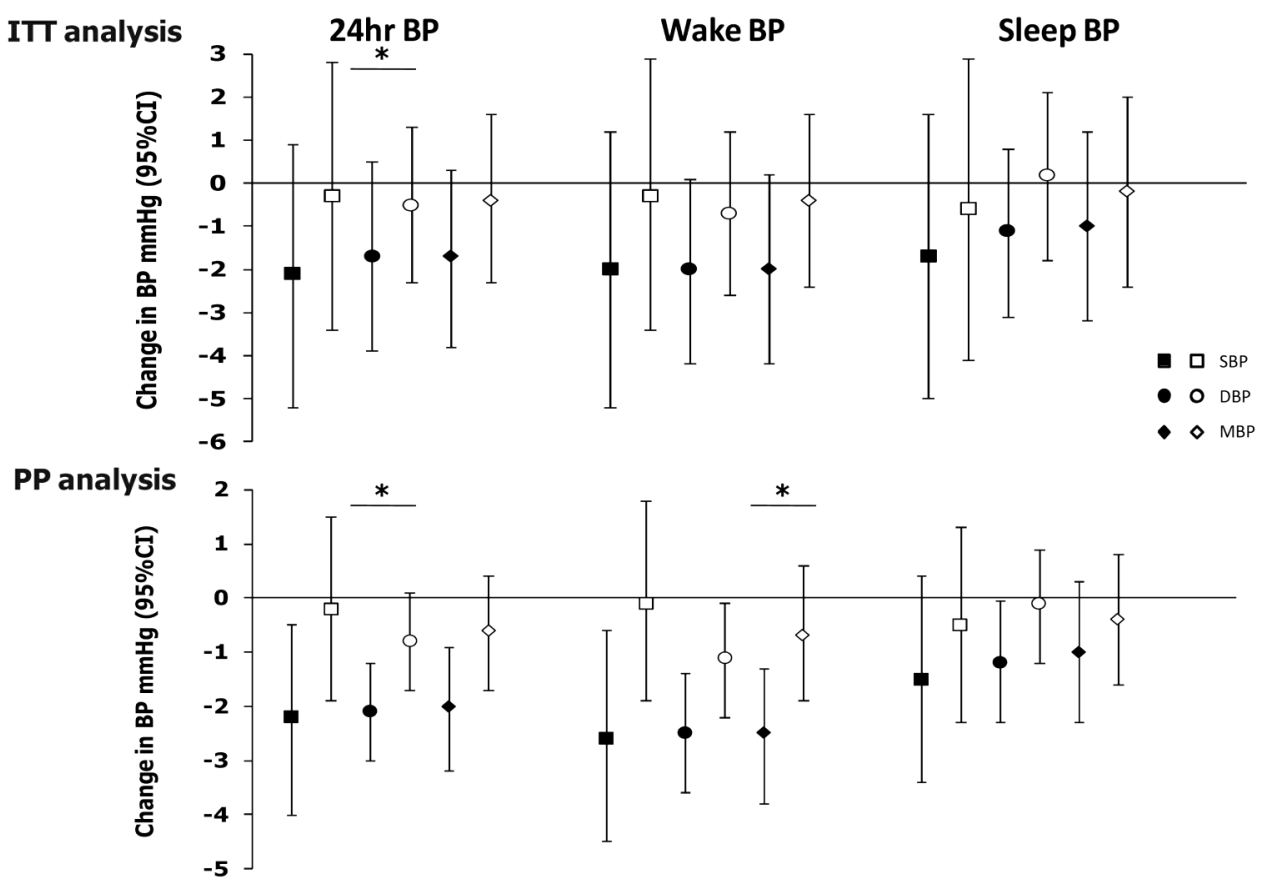

Figure 2 Change from baseline in $24 \mathrm{~h}$ blood pressure variables. Data represent mean differences from baseline (95\% Cl) on FP-CPAP (closed symbols) and AutoCPAP (open symbols) for the $24 \mathrm{~h}$, Wake and Sleep periods. Top panels: Intention-to-treat (ITT) analysis. Bottom panels: Per-protocol (PP) analysis. " $\mathrm{p}<0.05$. BP, blood pressure; DBP, diastolic blood pressure; MBP, mean blood pressure; SBP, systolic blood pressure. 
become standard in many European sleep centres. ${ }^{48}$ Moreover, neither the index of residual events nor compliance differed between the two arms. The expected decrease of $6 \mathrm{~mm} \mathrm{Hg}$ in SBP in the fixed-pressure arm based on the preliminary data of Patruno et $a l^{22}$ was probably not realistic to achieve. The recent meta-analysis by Bratton et $a l^{17}$ shows that compared with an inactive control, CPAP was associated with a reduction in SBP of $2.5 \mathrm{~mm} \mathrm{Hg}(95 \%$ CI 1.5 to $3.5 \mathrm{~mm} \mathrm{Hg})$. This is exactly the effect size that we found in our FP-CPAP arm.

\section{Conclusions and perspectives}

Generally, the impact of CPAP in reducing BP is relatively modest even in patients with sleepy OSA. Our study did not demonstrate that FP-CPAP is more effective than AutoCPAP regarding the primary outcome of office BP. However, indicating FP-CPAP treatment for patients with OSA with high cardiovascular risk, such as those with poorly controlled or resistant hypertension, might be of interest as our study found a higher impact of FP-CPAP than AutoCPAP, on $24 \mathrm{~h}$ DBP (a secondary outcome).

Acknowledgements The authors thank Alison Foote (Grenoble Alpes University Hospital) for editing the language.

Contributors JLP led the study and wrote the first draft of the report. NA and JFT undertook the data analyses. All authors participated in design, execution and oversight of the study. All authors had access to the data, commented on subsequent drafts and approved the final submitted version. JLP will act as guarantor and made the final decision to submit the manuscript for publication.

Funding The study was funded by the "Fondation Agir pour les maladies chroniques". The corresponding author had full access to all the data in the study and had final responsibility for the decision to submit for publication.

Competing interests None declared.

Patient consent Obtained.

Ethics approval Comité de Protection des Personnes Sud-Est V.

Provenance and peer review Not commissioned; externally peer reviewed.

\section{REFERENCES}

1 Young T, Palta M, Dempsey J, et al. The occurrence of sleep-disordered breathing among middle-aged adults. N Engl J Med 1993;328:1230-5.

2 Young T, Peppard PE, Gottlieb DJ. Epidemiology of obstructive sleep apnea: a population health perspective. Am J Respir Crit Care Med 2002;165:1217-39.

3 Peppard PE, Young T, Palta M, et al. Prospective study of the association between sleep-disordered breathing and hypertension. N Engl J Med 2000;342:1378-84.

4 Baguet JP, Barone-Rochette G, Tamisier $R$, et al. Mechanisms of cardiac dysfunction in obstructive sleep apnea. Nat Rev Cardiol 2012;9:679-88.

5 Marin JM, Carrizo SJ, Vicente E, et al. Long-term cardiovascular outcomes in men with obstructive sleep apnoea-hypopnoea with or without treatment with continuous positive airway pressure: an observational study. Lancet 2005; 365:1046-53.

6 Yaggi HK, Concato J, Kernan WN, et al. Obstructive sleep apnea as a risk factor for stroke and death. N Engl J Med 2005;353:2034-41.

7 Gami AS, Olson EJ, Shen WK, et al. Obstructive sleep apnea and the risk of sudden cardiac death: a longitudinal study of 10,701 adults. J Am Coll Cardiol 2013;62:610-16

8 Patel SR, White DP, Malhotra A, et al. Continuous positive airway pressure therapy for treating sleepiness in a diverse population with obstructive sleep apnea: results of a meta-analysis. Arch Intern Med 2003;163:565-71.

9 Giles TL, Lasserson TJ, Smith BJ, et al. Continuous positive airways pressure for obstructive sleep apnoea in adults. Cochrane Database Syst Rev 2006;(1): CD001106.

10 Marshall NS, Barnes M, Travier N, et al. Continuous positive airway pressure reduces daytime sleepiness in mild to moderate obstructive sleep apnoea: a meta-analysis. Thorax 2006;61:430-4.

11 Antic NA, Catcheside P, Buchan C, et al. The effect of CPAP in normalizing daytime sleepiness, quality of life, and neurocognitive function in patients with moderate to severe OSA. Sleep 2011:34:111-19.

12 Haentjens P, Van Meerhaeghe A, Moscariello A, et al. The impact of continuous positive airway pressure on blood pressure in patients with obstructive sleep apnea syndrome: evidence from a meta-analysis of placebo-controlled randomized trials. Arch Intern Med 2007;167:757-64
13 Bazzano LA, Khan Z, Reynolds K, et al. Effect of nocturnal nasal continuous positive airway pressure on blood pressure in obstructive sleep apnea. Hypertension 2007; $50: 417-23$

14 Alajmi M, Mulgrew AT, Fox J, et al. Impact of continuous positive airway pressure therapy on blood pressure in patients with obstructive sleep apnea hypopnea: a meta-analysis of randomized controlled trials. Lung 2007;185:67-72.

15 Montesi SB, Edwards BA, Malhotra A, et al. The effect of continuous positive airway pressure treatment on blood pressure: a systematic review and meta-analysis of randomized controlled trials. J Clin Sleep Med 2012;8:587-96.

16 Bakker JP, Edwards BA, Gautam SP, et al. Blood pressure improvement with continuous positive airway pressure is independent of obstructive sleep apnea severity. J Clin Sleep Med 2014;10:365-9.

17 Bratton DJ, Gaisl T, Wons AM, et al. CPAP vs mandibular advancement devices and blood pressure in patients with obstructive sleep apnea: a systematic review and meta-analysis. JAMA 2015;314:2280-93.

18 Martínez-García MA, Capote F, Campos-Rodríguez F, et al. Effect of CPAP on blood pressure in patients with obstructive sleep apnea and resistant hypertension: the HIPARCO randomized clinical trial. JAMA 2013;310:2407-15.

19 Barbé F, Durán-Cantolla J, Sánchez-de-la-Torre M, et al. Effect of continuous positive airway pressure on the incidence of hypertension and cardiovascular events in nonsleepy patients with obstructive sleep apnea: a randomized controlled trial. JAMA 2012;307:2161-8.

20 Bratton DJ, Stradling JR, Barbé F, et al. Effect of CPAP on blood pressure in patients with minimally symptomatic obstructive sleep apnoea: a meta-analysis using individual patient data from four randomised controlled trials. Thorax 2014:69:1128-35.

21 Pépin JL, Timsit JF, Tamisier R, et al. Is CPAP effective in reducing blood pressure in minimally symptomatic obstructive sleep apnoea? Thorax 2014;69:1068-70.

22 Patruno V, Aiolfi S, Costantino G, et al. Fixed and autoadjusting continuous positive airway pressure treatments are not similar in reducing cardiovascular risk factors in patients with obstructive sleep apnea. Chest 2007;131:1393-9.

23 Sanders $\mathrm{MH}$, Montserrat JM, Farré $\mathrm{R}$, et al. Positive pressure therapy: a perspective on evidence-based outcomes and methods of application. Proc Am Thorac Soc 2008:5:161-72.

24 Fuchs FS, Wiest GH, Frank M, et al. Auto-CPAP therapy for obstructive sleep apnea: induction of microarousals by automatic variations of CPAP pressure? Sleep 2002;25:514-18

25 Marrone 0 , Insalaco G, Bonsignore MR, et al. Sleep structure correlates of continuous positive airway pressure variations during application of an autotitrating continuous positive airway pressure machine in patients with obstructive sleep apnea syndrome. Chest 2002;121:759-67.

26 Hertegonne K, Bauters F. The value of auto-adjustable CPAP devices in pressure titration and treatment of patients with obstructive sleep apnea syndrome. Sleep Med Rev 2010;14:115-19.

27 Berry RB, Budhiraja R, Gottlieb DJ, et al. Rules for scoring respiratory events in sleep: update of the 2007 AASM Manual for the Scoring of Sleep and Associated Events. Deliberations of the Sleep Apnea Definitions Task Force of the American Academy of Sleep Medicine. J Clin Sleep Med 2012:8:597-619.

28 Mancia G, De Backer G, Dominiczak A, et al. 2007 Guidelines for the Management of Arterial Hypertension: The Task Force for the Management of Arterial Hypertension of the European Society of Hypertension (ESH) and of the European Society of Cardiology (ESC). J Hypertens 2007;25:1105-87.

29 Vivodtzev I, Tamisier R, Baguet JP, et al. Arterial stiffness in COPD. Chest 2014;145:861-75.

30 Schulz KF, Grimes DA. Multiplicity in randomised trials II: subgroup and interim analyses. Lancet 2005;365:1657-61.

31 Rubin DB. Multiple imputation for nonresponse in surveys. John Wiley \& Sons, 2004.

32 Rubin DB. Multiple imputation after 18+ years. J Am Stat Assoc 1996;91:473-89.

33 Kearney PM, Whelton M, Reynolds K, et al. Global burden of hypertension: analysis of worldwide data. Lancet 2005;365:217-23.

34 Lawes CM, Vander Hoorn S, Rodgers A. Global burden of blood-pressure-related disease, 2001. Lancet 2008;371:1513-18.

35 Marin JM, Agusti A, Villar I, et al. Association between treated and untreated obstructive sleep apnea and risk of hypertension. JAMA 2012;307:2169-76.

36 Nolan GM, Doherty LS, Mc Nicholas WT. Auto-adjusting versus fixed positive pressure therapy in mild to moderate obstructive sleep apnoea. Sleep 2007;30:189-94.

37 West SD, Nicoll DJ, Stradling JR. Prevalence of obstructive sleep apnoea in men with type 2 diabetes. Thorax 2006;61:945-50.

38 West SD, Jones DR, Stradling JR. Comparison of three ways to determine and deliver pressure during nasal CPAP therapy for obstructive sleep apnoea. Thorax 2006:61:226-31.

39 Ip S, D'Ambrosio C, Patel K, et al. Auto-titrating versus fixed continuous positive airway pressure for the treatment of obstructive sleep apnea: a systematic review with meta-analyses. Syst Rev 2012;1:20.

40 Turnbull F. Effects of different blood-pressure-lowering regimens on major cardiovascular events: results of prospectively-designed overviews of randomised trials. Lancet 2003;362:1527-35. 
41 Chobanian AV, Bakris GL, Black HR, et al. The Seventh Report of the Joint National Committee on Prevention, Detection, Evaluation, and Treatment of High Blood Pressure: the JNC 7 report. JAMA 2003;289: 2560-72.

42 Pepin JL, Borel AL, Tamisier R, et al. Hypertension and sleep: overview of a tight relationship. Sleep Med Rev 2014;18:509-19.

43 Pépin JL, Tamisier R, Barone-Rochette G, et al. Comparison of continuous positive airway pressure and valsartan in hypertensive patients with sleep apnea. Am J Respir Crit Care Med 2010;182:954-60.

44 Thunström E, Manhem $\mathrm{K}$, Rosengren $\mathrm{A}$, et al. Blood pressure response to losartan and continuous positive airway pressure in hypertension and obstructive sleep apnea. Am J Respir Crit Care Med 2016;193:310-20.
45 Mwenge GB, Dury M, Delguste $P$, et al. Response of automatic continuous positive airway pressure devices in a normal subject. Eur Respir J 2011;37:1530-3.

46 Karasulu L, Epöztürk PO, Sökücü SN, et al. Improving Heart rate variability in sleep apnea patients: differences in treatment with auto-titrating positive airway pressure (APAP) versus conventional CPAP. Lung 2010;188:315-20.

47 Patruno V, Tobaldini E, Bianchi AM, et al. Acute effects of autoadjusting and fixed continuous positive airway pressure treatments on cardiorespiratory coupling in obese patients with obstructive sleep apnea. Eur J Intern Med 2014;25:164-8.

48 Craig SE, Kohler M, Nicoll D, et al. Continuous Positive Airway Pressure (CPAP) improves sleepiness but not calculated vascular risk in patients with minimally symptomatic obstructive sleep apnoea (OSA); the MOSAIC randomised controlled trial. Thorax 2012;67:1090-6. 